We performed below the IBD audit for:

- Sigmoidoscopy in 72 hours (28\% vs 99\%)

- Prescribing $\mathrm{Ca} /$ vit D (65\% vs $74 \%)$

- Median time to surgery (9 vs 7.5 days)

Important standards of IBD nurse and dietician review maintained. Delay in endoscopic evaluation and therefore time to surgery indicate there has been a slipping of standards in ASC care. This may be related to less direct ward continuity.

Our data show a drop in performance (access to endoscopy and time to surgery). They have allowed us to critically appraise our acute IBD service thus leading to care delivery change and an education package for medical and surgical directorates. A repeat audit is planned in 24 months to demonstrate quality improvement as a result of this.

\section{PWE-044 THE IBD BIORESOURCE: PROGRESSING FROM GENETICS TO FUNCTION AND CLINICAL TRANSLATION IN CD \& UC}

\footnotetext{
1,2 Laetitia Pele* ${ }^{*}$ 1,2Rachel Simpkins, ${ }^{1,2}$ Catherine Thorbinson, 1,2Deepthy Francis, 1,2Sophie Lewis, ${ }^{2}$ Rasha Shawky, ${ }^{1,2}$ Miles Parkes, ${ }^{1,2}$ UK IBD Genetic Consortium. ${ }^{1}$ Department of Medicine, University of Cambridge, UK, Cambridge, UK; ${ }^{2}$ IBD Bioresource, NIHR BioResource, Cambridge, UK, Cambridge, UK
}

\subsection{6/gutjnl-2018-BSGAbstracts. 176}

Introduction The Inflammatory Bowel Disease (IBD) BioResource was established by the UK IBD Genetics Consortium and the NIHR BioResource in 2016 to expedite the clinical translation of recent genetics advances and support important IBD research. It recruits patients from hospitals UK-wide, the aim being to enlist 25000 participants who can be further invited for future research studies based on genotype and/or phenotype. DNA samples will also be used in on-going IBD genetics analyses by the UK IBD Genetics Consortium.

Methods The Main cohort comprises individuals with established Crohn's Disease (CD) and Ulcerative Colitis (UC). Both clinical and self-reported phenotype data are collected, alongside plasma, serum and DNA samples for whole Genome Sequencing. The Inception cohort aims to recruit a sub-set of 1000 individuals newly diagnosed with IBD that will provide more detailed sampling, unconfounded by drug treatment or effects of surgery and includes stool, biopsy tissue and whole blood for RNA. This cohort offers a unique resource to undertake transcriptomic, meta-genomic, metabolomic and proteomic studies and facilitate research into determinants, predictors and biomarkers of IBD disease course and treatment response. In addition to Stage 1 recruitment, the IBD BioResource panel can be accessed by any researchers (both academic and commercial) with ethically approved proposals and may involve a range of possible options, such as access to data or samples or recall of genotype-selected participants to donate further samples or trial novel therapies.

Results 24 months in, we have 59 hospitals active and $\sim 30$ in set-up.

Main Cohort: over 8000 patients have been recruited - CD/ UC 4,390/3,832.

CD phenotypes: extent - 31\% ileal, 32\% ileo-colonic, 31\% colonic, $28 \%$ with peri-anal involvement; behaviour - $68 \%$ inflammatory, $21 \%$ stenosing and $11 \%$ penetrating.

$5048(61 \%)$ have been prescribed thiopurines and 2324 $(28 \%)$ required treatment cessation. $3842(47 \%)$ have received anti-TNF $\alpha$ therapy.
45\% CD and 5\% UC have required surgery. Most subjects are recruited in medical gastroenterology clinics so there are biases in this dataset. We will provide updates and breakdown of numbers at the meeting and discuss downstream stage 2 studies.

Inception Cohort: has been launched and is being rolled out nationally.

Conclusion The IBD BioResource and its network are on course to generate an accessible platform of patients and their data that will facilitate high quality translational IBD research.

\section{PWE-045 VEDOLIZUMAB RESULTS IN REDUCED HOSPITALISATION AND STEROID USE OVER 1-YEAR: RESULTS FROM THE SCOTTISH VEDOLIZUMAB COHORT}

${ }^{1}$ Nikolas Plevris*, ${ }^{1}$ Cher Chuah, ${ }^{1}$ Phil Jenkinson, ${ }^{2} \mathrm{R}$ Allen, ${ }^{2} \mathrm{P}$ Baker, ${ }^{3}$ Paul Brennan, ${ }^{1}$ Antonia Churchhouse, ${ }^{1} S$ Din, ${ }^{4}$ Emma Donoghue, ${ }^{2}$ Daniel Gaya, ${ }^{3}$ Max Groome, ${ }^{5} \mathrm{H}$ Jafferbhoy, ${ }^{1} \mathrm{G}$ Jones, ${ }^{2}$ Jonathan Macdonald, ${ }^{2}$ Morag MacMaster, ${ }^{3} \mathrm{Craig}$ Mowat, ${ }^{2} \mathrm{G}$ Naismith, ${ }^{1} \mathrm{C}$ Noble, ${ }^{6} \mathrm{~L}$ Potts, ${ }^{2} \mathrm{E}$ Saffouri, ${ }^{2} \mathrm{JP}$ Seenan, ${ }^{5} \mathrm{~A}$ Sengupta, ${ }^{1} \mathrm{Alan}$ Shand, ${ }^{3} \mathrm{P}$ Shasi, ${ }^{3} \mathrm{~J}$ Todd, ${ }^{2} \mathrm{~J}$ Veryan, ${ }^{6} \mathrm{~A}$ Watson, ${ }^{1} \mathrm{E}$ Watson, ${ }^{4} \mathrm{D}$ Watts, ${ }^{1} \mathrm{I}$ Arnott, ${ }^{1} \mathrm{C}$ Lees. ${ }^{1}$ The Edinburgh IBD Unit, Western General Hospital, Edinburgh, UK; ${ }^{2}$ NHS Greater Glasgow and Clyde, Glasgow, UK; ${ }^{3}$ NHS Tayside, Dundee, UK; ${ }^{4}$ NHS Forth Valley, Forth Valley, UK; ${ }^{5}$ NHS Fife, Kirkcaldy, UK; ${ }^{6}$ NHS Highlands, Inverness, UK

\subsection{6/gutjnl-2018-BSGAbstracts. 177}

Introduction The GEMINI trials and an increasing body of real-world data have demonstrated the effectiveness and safety of vedolizumab (VDZ) in IBD. However, there is limited available data about its effect on hospitalisations and steroid use. Our aim was to address this in a large real-world cohort of IBD patients from across Scotland.

Methods A multicenter retrospective cohort analysis of medical records was performed across 7 Scottish healthcare trusts. Primary outcomes were hospitalisation rates and overall steroid use in patients remaining on VDZ. Secondary outcomes were safety and intention to treat steroid free remission rates in patients with active disease. All data were prospectively collected as part of routine clinical care. Baseline demographics, clinical scores (HBI or Partial Mayo), faecal calprotectin (FC), endoscopy and radiology at 3, 6 and 12 months was recorded where available. Active disease was defined as endoscopic or radiographic evidence of disease or FC $>200 \mathrm{mcg} / \mathrm{g}$. Clinical remission was defined as $\mathrm{HBI}<5$ or Partial Mayo $<2$. Biochemical remission was defined as $\mathrm{FC}<200 \mathrm{mcg} / \mathrm{g}$.

Results 340 (137 UC and 203 CD) patients were included in the primary analysis with a median follow-up of 9.4 months. Hospitalisation rates per patient-year were $0.60,0.67,0.36$ and 0.16 at baseline, 3,6 and 12 months of treatment respectively. Total number of hospitalisations reduced by $52.5 \%$ from 204 (12 months prior to VDZ) to 97 (12 months after VDZ). Proportion of patients on concomitant steroids reduced from $39.7 \%$ to $16.7 \% \quad(n=332), \quad 8.1 \% \quad(n=270), \quad 9.3 \%$ $(\mathrm{n}=194)$ at 3,6 and 12 months respectively. In patients with active CD $(n=153,75.4 \%)$ steroid free clinical and steroid free biochemical remission rates were; $54.4 \%$ and $30.2 \%$ at 3 months; $47.7 \%$ and $32.1 \%$ at 6 months; $28.6 \%$ and $33.9 \%$ at 12 months. In patients with active UC $(n=112,81.8 \%)$ steroid free clinical and steroid free biochemical remission rates were; $57.4 \%$ and $40.9 \%$ at 3 months; $51.6 \%$ and $39.1 \%$ at 6 months; $37.5 \%$ and $41.2 \%$ at 12 months. Our cohort received >2066 VDZ infusions, 2 (0.6\%) patients developed infusion reactions, $9(2.6 \%)$ patients developed serious infections and $17(5.0 \%)$ serious adverse events. 